\title{
Artificial Neural Network For Diagnosis OF PANCREATIC CANCER
}

\author{
Sanoob M.U ${ }^{1}$, Anand Madhu' ${ }^{2}$, Ajesh K.R ${ }^{3}$ and Surekha Mariam Varghese ${ }^{4}$ \\ Department of Computer Science and Engineering, Mar Athanasius College of \\ Engineering, Kothamangalam, Kerala
}

\begin{abstract}
Cancer is malignant growth or tumour which forms due to an uncontrolled division of cells in a part of body which may even lead to death. These are of different types depending upon the part of body affected. If it is Pancreas then the disease is termed as Pancreatic Cancer. This paper presents an Artificial Neural Network model to diagnose pancreatic cancer based on a set of symptoms. An ANN model is created after analysing the actual procedure of disease diagnosis by the doctor. An approach to detect various stages of cancer affected in pancreas is presented in the paper. Results of the study suggest the advantage of using ANN model instead of manual disease diagnosis.
\end{abstract}

\section{KEYWORDS}

Neural Network, Diagnosis, Fuzzy Logic, Cancer, Pancreatic Cancer

\section{INTRODUCTION}

Artificial Neural Network (ANN) is a relatively raw model based on the brain's neural structure. In various clinical situations which are considered difficult, ANN has been used successfully as a non-linear pattern recognition technique in making diagnostic and prognostic decisions [1]. Now a days, many medical diagnosis problems are being solved using NN techniques. Artificial Neural Networks are applied to medicine mainly for the task which is based on the measured features to assign the patient to one of a small set of classes [2][3]. A number of researches are going on worldwide on the applicability of neural networks in medical diagnosis [4][5]. Accuracy as well as the objectivity of medical diagnosis has been increased using neural networks. In ANN, the processing element is called as neurons. An artificial Neural Network is a network of such interconnected neurons operating in parallel. Biological nervous systems are the main inspiration behind the concept of artificial neurons. Functioning of a network greatly depends on the connection between the elements in the network. These processing elements are subdivided into several subgroups called layers in the network. The first and the last layers are called the input and output layers respectively. There may be some additional layers, called hidden layers, in between the input and output layers. A neural network can be trained to perform a particular functionality. This is done by adjusting the values of the connections between the elements, called weights. The number of cancer related deaths worldwide is increasing day by day and researches shows that pancreatic cancer is the eighth most common cause of cancer-related deaths worldwide and fourth worldwide.

Malignant type neoplasm pancreatic cancer is originated from transformed cells which arise in tissues form the pancreas. Pancreas is a spongy organ that is around 6-inch long. This is located

DOI: $10.5121 /$ ijci.2016.5205 
in the back of the abdomen behind the stomach. Pancreatic juices, insulin, and hormones are created by exocrine and endocrine glands. These glands are contained within the pancreas. The exocrine glands make the enzymes or pancreatic juices, which are then released to the intestines through a series of ducts. This will help carbohydrates, proteins and fat to digest. Islets of Langerhans are the small clusters of these endocrine cells. The glucagon and insulin are released into the bloodstream by these islets of Langerhans. The levels of sugar in blood are managed by two of these hormones. Improper working of these hormones will often result in diabetes. Tumours are formed by the abnormal pancreas tissues continue splitting and create masses or lumps of tissues. The major functions of pancreas are then interfered by these tumours. Benign is the situation when a tumour stays at one location and shows limited growth.

This paper tried to show that if the particular condition of a patient is given, then the NN can be used to make an accurate prognosis of each individual [6] [7]. How human intelligence can be applied in health sector [8] [9] is the major concern behind this paper. A self-learning intelligent system can be developed using $\mathrm{NN}$ which can overcome the uncertainties in the diagnosis of pancreatic cancer. Some symptoms are taken from the patient's previous medical records as well as from the doctor, and by using these data the neural network model is trained to detect the presence/absence of pancreatic cancer in that patient. To diagnose the pancreatic cancer properly using this intelligent model fuzzified symptoms values are applied.

In prediction, NN models are widely being used, especially in medical diagnosis. Studies show that for the diagnosis of different medical diseases, ANN have been used very successfully. In 2004, a NN based model is proposed by Kamruzzaman et al. for the diagnosis of heart diseases [10]. In 2008, a Genetic Algorithm (GA) based technique for classifying tumour mass in breast and to identify breast cancer has been introduced [11]. A new method for Predicting Blood Cancer and Disorder is then developed by Payandeh [12] et al. later. One of the latest works in this is Artificial Neural Network for predicting headache which is done by Bahar et al. In 2011 also, a lot of $\mathrm{NN}$ based disease diagnosis has been done. Artificial Neural Network to prediagnosis of Hypertension [13], using Back-Propagation training algorithm, Artificial Neural Network model to diagnose skin diseases by Backpo [14] et al. etc are some of them. Similarly ANN models are also developed for breast cancer detection [15], Kidney stone diseases [16] etc. In the following sections the paper will be dealing with the details of implementation of the ANN model for detecting the pancreatic cancer. In section II, the Methodology used to in the paper is discussed. The results of the experiment and Discussions are included in Section III. Then by Section IV, the paper is concluded.

\section{Methodology}

The initial step towards performing the process of medical diagnosis was initiated by examining a number of patients by a group of medical experts and identifying the symptoms. The next step was to propose a neural network which could be used in diagnosing PC diseases. The proposed model contains three layers namely input layer, hidden layer, and output layer. A single hidden layer consisting of 20 hidden layer neurons was created and trained. The input samples and output or target samples were divided for training, validation and test sets automatically.

For training the network, the training set was made use of. Training was continued until there was network stops improving the validation set. The test set was completely independent of the 
measure of network accuracy. During the training phase, the patterns in data were learned by hidden neurons and the relationship between input and output pairs are mapped. In the hidden layer, a transfer function was used by each neuron for processing the data it receives from input layer and the processed information to the output neurons was transferred, for further processing using a transfer function in each neuron.

\subsection{DATASET}

Dataset used for the diagnosis of the pancreatic cancer is shown in Table 1. The data set consist of 11 possible symptoms and 3 outcomes possible. The outcome is purely dependent on the significance of symptoms for a particular patient. The entire dataset consists of measured features of 120 patients in which 90 samples were used for training the network and the remaining for testing purpose.

The set of symptoms represented as $\mathrm{S}=\{$ Jaundice (J), Loss of Appetite (LA), Weight Loss (WL), Pain in Upper Abdomen (PUA), Irritability (I), Gall Bladder Enlargement (GBE), Swelling Lymph (SL), Diabetes Mellitus (DM), Deep Venous Thrombosis (DVT), Acholic Stool \&Steatorrhea (AS\&S) and Fatty Tissue Abnormalities (FTA) \}.

The set of possible outcomes of diagnosis represented as $\mathrm{D}=\{$ Disease Detected, Disease might be Detected and Disease not Detected $\}$.

Disease outcomes and the corresponding label assigned for each of them is explained in Table 2 . Next, on the basis of fuzzy set, the paper describes each symptom by its membership value. The basic block diagram that explains about the operational procedure is shown in Figure. 1.

Table 1. Symptoms Significance and Result

\begin{tabular}{|c|c|c|c|c|c|c|c|c|c|c|c|}
\hline J & LA & WL & PUA & I & GBE & SL & DM & DVT & AS\&S & FTA & Remarks \\
\hline 0.45 & 0.26 & 0.60 & 0.80 & 0.30 & 0.10 & 0.35 & 0.15 & 0.55 & 0.72 & 0.18 & 1 \\
\hline 0.18 & 0.00 & 0.62 & 0.59 & 0.17 & 0.78 & 0.82 & 0.50 & 0.14 & 0.36 & 0.47 & 2 \\
\hline 0.73 & 0.69 & 0.32 & 0.33 & 0.25 & 0.55 & 0.13 & 0.20 & 0.61 & 0.49 & 0.86 & 2 \\
\hline 0.24 & 0.63 & 0.28 & 0.08 & 0.39 & 0.36 & 0.70 & 0.55 & 0.23 & 0.17 & 0.63 & 3 \\
\hline 0.59 & 0.68 & 0.43 & 0.75 & 0.73 & 0.29 & 0.37 & 0.13 & 0.70 & 0.55 & 0.43 & 1 \\
\hline 0.44 & 0.53 & 0.56 & 0.69 & 0.57 & 0.63 & 0.19 & 0.41 & 0.34 & 0.72 & 0.38 & 1 \\
\hline 0.52 & 0.63 & 0.72 & 0.30 & 0.60 & 0.19 & 0.40 & 0.21 & 0.42 & 0.39 & 0.52 & 3 \\
\hline 0.13 & 0.12 & 0.63 & 0.24 & 0.11 & 0.47 & 0.23 & 0.51 & 0.69 & 0.10 & 0.63 & 2 \\
\hline 0.63 & 0.38 & 0.33 & 0.21 & 0.49 & 0.72 & 0.62 & 0.24 & 0.77 & 0.43 & 0.40 & 1 \\
\hline 0.24 & 0.55 & 0.78 & 0.30 & 0.54 & 0.41 & 0.78 & 0.43 & 0.64 & 0.18 & 0.23 & 1 \\
\hline
\end{tabular}

Table 2. Assigned Labels and Outcome

\begin{tabular}{|c|c|}
\hline Label & Outcome \\
\hline 1 & Detected \\
\hline 2 & Might be Detected \\
\hline 3 & Not Detected \\
\hline
\end{tabular}




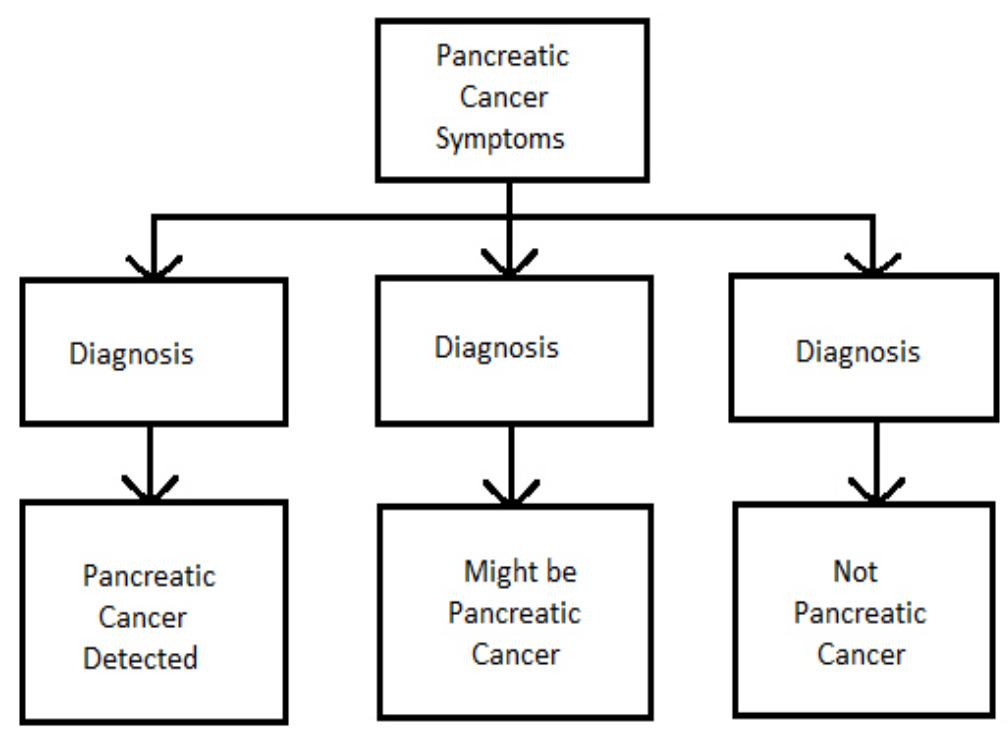

Figure 1. Operational Procedure

\subsection{Training of Parameters}

The network will become ready to be trained, only once that network is organized and structured for the targeted application. The initial weight has to be chosen at random for starting this process. Training will be started after that. The network is trained by using existing set of data which is directly obtained from various patients and whose output is well known. The neurons in hidden layer will learn the data pattern while training and map relation between input pairs and output pairs. Each hidden layer neuron made use of a transfer function for processing data that accepts from input layer and transfers the information which is processed to the output neurons for continuing the processing in each neuron using a transfer function.

\section{RESULTS AND DiSCUSSION}

Matlab R2011a's toolbox for Neural network is used for performance evaluation for the new networks which consists of a 11 number three layer feed forward network of inputs and sigmoid hidden neurons and linear output neurons is suggested. The new approach used LevenbergMarquardt algorithm for back propagation for training the network where training stops automatically when generalization stops improving, as indicated by an increase in the MSE (Mean Square Error) of the samples used for validation. The proposed neural network is shown in Figure. 2. 


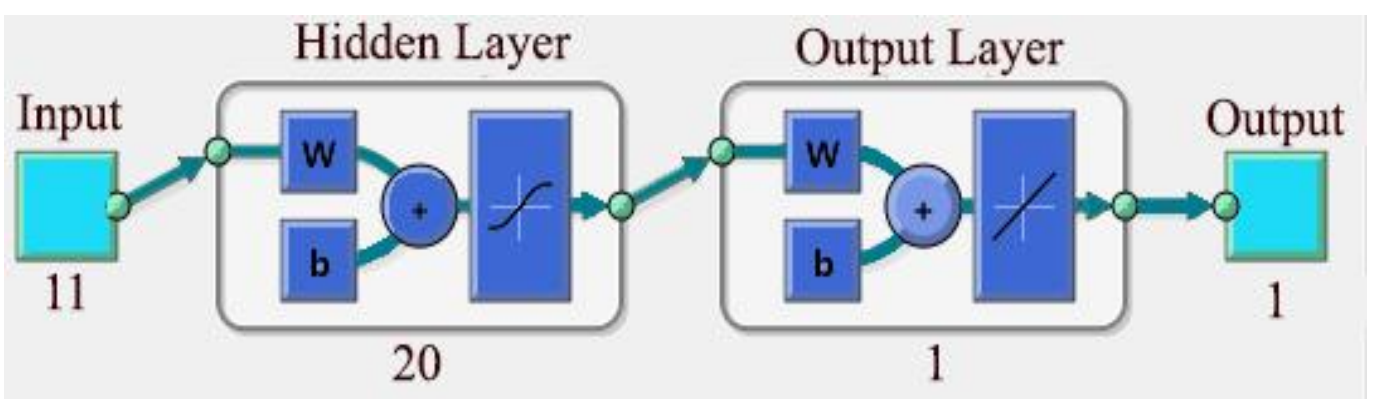

Figure 2. Proposed Neural Network

A membership based fuzzification scheme is adopted here for converting our dataset to to a fuzzified set of symptoms. After an interview with physicians, a linear membership function was again selected for each symptom. Three to five linguistic variables were assigned to each symptom normally, and then repeated the classification tests.

The experimental results of implementing the new ANN methodology to distinguish between Pancreatic Cancer affected and non-affected patients based upon specified symptoms represents good capabilities of the network to learn the training patterns corresponding to symptoms of the patients. The experimental setup is shown in Figure. 3 


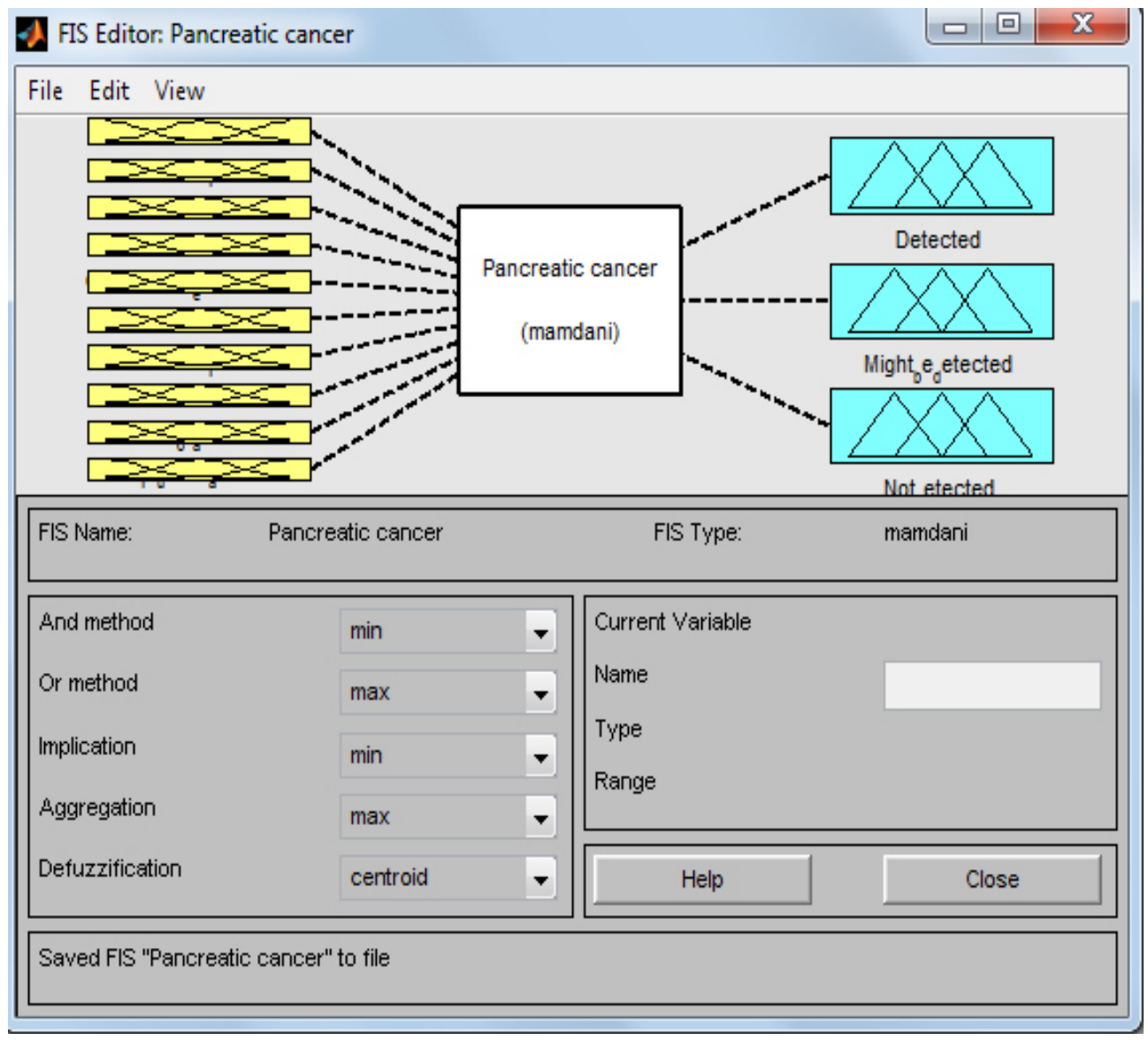

Figure 3. FIS Editor

A triangular membership function is used for fuzzifying the inputs. Using Matlab, a membership function editor is used. This Membership function editor is shown in the Figure. 4. 
International Journal on Cybernetics \& Informatics (IJCI) Vol. 5, No. 2, April 2016

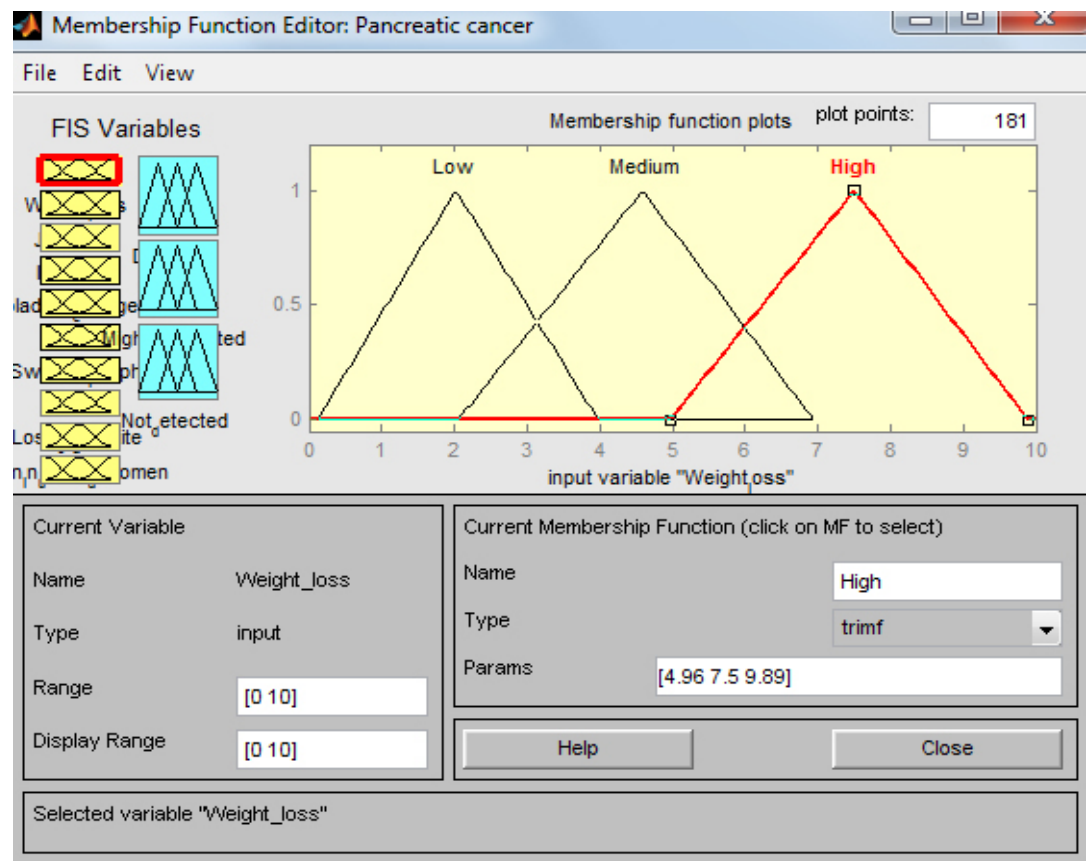

Figure 4. Membership Function Editor

The performance graph plotted based on the results obtained is shown in Figure. 5.

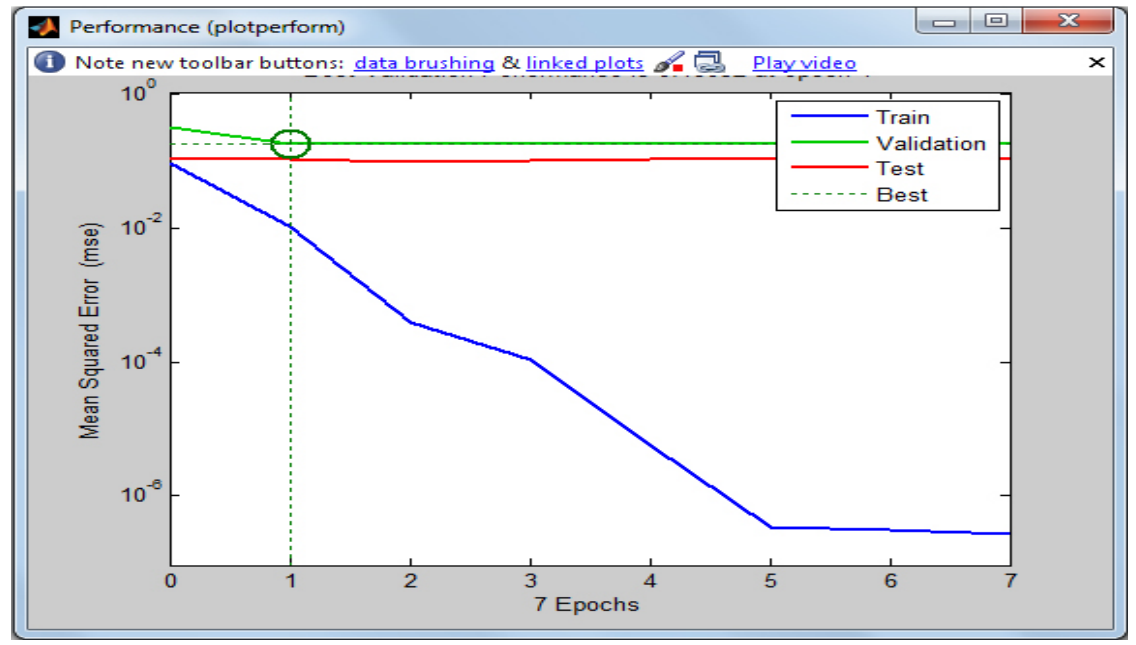

Figure 5. Performance Plot 
International Journal on Cybernetics \& Informatics (IJCI) Vol. 5, No. 2, April 2016

\section{Conclusion}

An approach for the diagnosis of out-of-controlled cell growth in pancreas based on Artificial Neural Network is explained in this paper. Detection of cancer in the pancreatic cells at its early stage is necessary for its better treatment and cure. Hence it is important to detect pancreatic cancer automatically to mitigate the real-world medical problems. Here we have presented how effectively we can made use of neural networks in the detection and diagnosis of cancer in pancreatic cells. The construction of a diagnostic system which is highly accurate based on neural network model is done here. Also an investigation on the performance of neural network structure is done in this paper. This model is designed in such a way that it made use of fuzzy values instead of normal values for the incorporation of the neural network context. The model has provision to accept symptoms in a patient and it will inform the present condition of that patient, based on the evaluation made on the input symptoms. So this model is an interactive model. So the early detection can be done and hence it will help the doctor to plan and do the better medication for the patient. The symptoms are fuzzified and network is implemented based on around 20 neurons, for getting the better performance and accurate diagnosis. Outcome of the experiments indicates that the noval approach is able to valuate data in most efficient way compared to other normal approaches. Future works can be extended for other similar disease detection comprising complex and related datasets with similar or better accuracy..

\section{REFERENCES}

[1] N.Salim, Medical Diagnosis Using Neural Networks, 2004.

[2] W. David Aha and Dennis Kibler, Instance-based prediction of heart disease presence with the Cleveland database

[3] J. W., Everhart, J. E., Dickson, W. C., Knowler, W. C., Johannes, R. S.,Using the ADAP learning algorithm to forecast the onset of diabetes mellitus, Proc. Symp. on Computer Applications and Medical Care, pp. 2615, 1988.

[4] SuvarnaMahavirPatil and R.R. Mudholkar, An Osteoarthritis classifier using back-propagation neural network,International Journal of Advances in Engineering \& Technology, Sept 2012, ISSN: 2231-1963.

[5] J. W., Everhart, J. E., Dickson, W. C., Knowler, W. C., Johannes, R.S.,Using the ADAP learning algorithm to forecast the onset of diabetes mellitus, Proc. Symp. on Computer Applications and Medical Care, pp. 2615, 1988.

[6] Imianvan Anthony Agboizebeta., and Obi Jonathan Chukwuyeni, Application of Neuro-Fuzzy Expert System for the Probe and Prognosis of Thyroid Disorder, International Journal of Fuzzy Logic Systems(IJFLS) Vol.2, No.2, April 2012.

[7] Obi J.C. Imianvan A.A, Interactive Neuro-Fuzzy Expert system for diagnosis of Luukemia, Global Journal of Computer Science and Technology, Volume 11 Issue 12 Version 1.0 July 2011.

[8] W. David Aha and Dennis Kibler, Instance-based prediction of heart disease presence with the Cleveland database, 3rd ed. Harlow, England: Addison-Wesley, 1999.

[9] Prof. A. Maithili, Dr. R. VasanthaKumari Mr. S. Rajamanickam, Neural Networks towards medical, ,International Journal of Modern EngineeringResearch (IJMER), Vol.1, Issue1, pp-57-64 ISSN: 2249-6645.

[10] S. M. Kamruzzaman, Ahmed RyadhHasan, Abu BakarSiddiquee and Md. EhsanulHoqueMazumder, Medical diagnosis using neural network,, ICECE 2004, 28-30 December 2004, Dhaka, Bangladesh 
[11] Arpita Das and Mahua Bhattacharya, GA based Neuro Fuzzy Techniques for breast cancer Identification,, 3rd ed. IEEE, 978-7695-3332-2/08, 2008. DOI: 10.1109/IMVIP.2008.19

[12] Payandeh M, MehrnoushAeinfar, VahidAeinfar, Mohsen Hayati, A New Method for Diagnosis and Predicting Blood Disorder and Cancer Using Artificial Intelligence, IJHOSCR, Vol. 3, No.4; 2009.

[13] B. Sumathi,Dr. A. Santhakumaran, Pre-Diagnosis of Hypertension Using Artificial Neural Network, Global Journal of Computer Science and Technology,Global Journal of Computer Science andTechnology Volume 11 Issue 2 Version 1.0 February 2011

[14] Bakpo, F. S. and Kabari, L. G, Diagnosing Skin Diseases Using an Artificial Neural Network DOI:10.5772/16232.

[15] BipulPandey, Tarun Jain, Vishal Kothari and Tarush Grover, Evolutionary Modular Neural Network Approach for Breast Cancer Diagnosis, IJCSI International Journal of Computer Science Issues. Vol.9, Issue 1, No 2, January 2012.

[16] Koushal Kumar, Abhishek, Artificial Neural Networks for diagnosis of kidney stones disease, I.J. Information Technology and Computer Science, 2012, 7, 20-25

[17] W. David Aha and Dennis Kibler, Instance-based prediction of heart disease presence with the Cleveland database, 3rd ed. Harlow, England: Addison-Wesley, 1999.

\section{AUTHORS}

Sanoob M.U. is currently pursuing M.Tech in Computer Science and Engineering in Mar Athanasius College of Engineering. He completed his B.Tech from AdiShankara Institute of Engineering and Technology, Kalady. His areas of research are Machine Learning and Databases.

Ajesh K.R. is currently pursuing M.Tech in Computer Science and Engineering in Mar Athanasius College of Engineering. He completed his B.Tech from AdiShankara Institute of Engineering and Technology, Kalady. His areas of research are Machine Learning and Image Processing.

AnandMadhu is currently pursuing M.Tech in Computer Science and Engineering in Mar Athanasius College of Engineering. He completed his B.Tech from University College of Engineering, Thodupuzha. His areas of research are Machine Learning and Data Mining.

Surekha Mariam Varghese is currently heading the Department of Computer Science and Engineering, M.A. College of Engineering, Kothamangalam, Kerala, India. She received her B-Tech Degree in Computer Science and Engineering in 1990 from College of Engineering, Trivandrum affiliated to Kerala University and M-Tech in Computer and Information Sciences from Cochin University of Science and Technology, Kochi in 1996. She obtained Ph.D in Computer Security from Cochin University of Science and Technology, Kochi in 2009. She has around 25 years of teaching and research experience in various institutions in India. Her research interests include Network Security, Database Management, Data Structures and Algorithms, Operating Systems, Machine Learning and Distributed Computing. She has published 17 papers in international journals and international conference
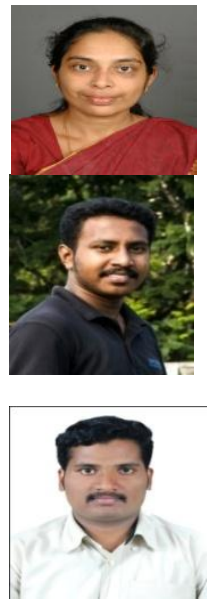
proceedings. She has been in the chair and reviewer for many international conferences and journals.

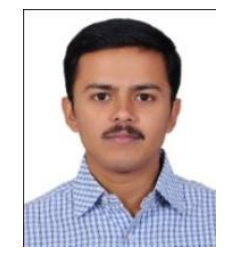

